Short Communication

\title{
The antimicrobial peptide TAT-RasGAP $317-326$ inhibits the formation and expansion of bacterial biofilms in vitro
}

\author{
Tytti Heinonen ${ }^{\mathrm{a}}$, Simone Hargraves ${ }^{\mathrm{a}}$, Maria Georgieva ${ }^{\mathrm{b}}$, Christian Widmann $^{\mathrm{b}}$, \\ Nicolas Jacquier ${ }^{\mathrm{a}, *}$ \\ a Institute of Microbiology, Lausanne University Hospital and University of Lausanne, Rue du Bugnon 48, 1011 Lausanne, Switzerland \\ ${ }^{\mathrm{b}}$ Department of Biomedical Sciences, University of Lausanne, Rue du Bugnon 7, 1005 Lausanne, Switzerland
}

\section{A R T I C L E I N F O}

\section{Article history:}

Received 23 December 2020

Revised 3 March 2021

Accepted 11 March 2021

Available online 20 April 2021

Editor: Prof Charles Kelly

\section{Keywords:}

Biofilm

Antimicrobial peptide

Acinetobacter baumannii

Pseudomonas aeruginosa

Staphylococcus aureus

TAT-RasGAP $317-326$

\begin{abstract}
A B S T R A C T
Objectives: Biofilms are structured aggregates of bacteria embedded in a self-produced matrix that develop in diverse ecological niches. Pathogenic bacteria can form biofilms on surfaces and in tissues, causing nosocomial and chronic infections that are difficult to treat. While antibiotics are largely inefficient in limiting biofilm formation and expansion, antimicrobial peptides (AMPs) are emerging as alternative antibiofilm treatments. In this study, we explore the effect of the newly described AMP TAT-RasGAP ${ }_{317-326}$ on Acinetobacter baumannii, Pseudomonas aeruginosa and Staphylococcus aureus biofilms.
\end{abstract}

Methods: Efficiency of TAT-RasGAP ${ }_{317-326}$ on biofilms was tested in vitro. Both viability of bacteria contained in the biofilm as well as biomass of the biofilm were quantified using resazurin and crystal violet staining, respectively. The antibiofilm effect of TAT-RasGAP $317-326$ was compared with a selection of classical antibiotics and AMPs.

Results: We observe that TAT-RasGAP ${ }_{317-326}$ inhibits biofilm formation at concentrations equivalent or two times greater than the minimum inhibitory concentration (MIC) of planktonic bacteria. Moreover, TAT-RasGAP ${ }_{317-326}$ limits the expansion of A. baumannii and $P$. aeruginosa established biofilms at twice the concentration inhibiting biofilm formation.

Conclusion: These results underscore the potential use of TAT-RasGAP ${ }_{317-326}$ against biofilms and encourage further studies in the development of AMPs to treat biofilm-related infections.

(c) 2021 The Author(s). Published by Elsevier Ltd on behalf of International Society for Antimicrobial Chemotherapy. This is an open access article under the CC BY-NC-ND license (http://creativecommons.org/licenses/by-nc-nd/4.0/)

\section{Introduction}

The emergence of antibiotic resistance is a major threat to public health. Infections caused by multidrug-resistant (MDR) bacteria are challenging to treat and lead to disability and even death [1]. One limitation in the development of novel antibiotics is the use of free-living (also called planktonic) bacteria in axenic medium as a model system. This model is not representative of infections, which are commonly caused by bacterial aggregates embedded in a selfproduced matrix, called biofilms [2].

\footnotetext{
* Corresponding author. Mailing address: Institute of Microbiology, Department of Pathology and Laboratory Medicine, Lausanne University Hospital and University of Lausanne, Rue du Bugnon 48, CH-1011 Lausanne, Switzerland. Tel.: +41 21314 8539.

E-mail address: Nicolas.Jacquier@chuv.ch (N. Jacquier).
}

Pathogenic bacteria, including Acinetobacter baumannii, Pseudomonas aeruginosa and Staphylococcus aureus, can form biofilms on medical devices implanted in humans, in some tissues (e.g. lungs, teeth and skin) and on healthcare surfaces [3]. The reversible formation of biofilms is triggered by signals such as nutrient limitation, antibiotic exposure and oxygen availability [2]. Bacteria secrete polysaccharides, DNA and proteins that compose the biofilm matrix. The latter forms a scaffold for bacterial attachment and protects bacteria from external insults. Bacteria embedded in biofilm develop into heterogeneous bacterial subpopulations that strongly differ from planktonic bacteria regarding both their gene expression profile and their functional properties [2]. The combination of intrinsic bacterial antibiotic resistance and resistance provided by the biofilm structure renders biofilms highly challenging to eradicate. Thus, we need alternatives to classical antibiotics to treat biofilms, especially when containing MDR pathogens. 
Antimicrobial peptides (AMPs) are short peptides first described as a defence mechanism of living organisms towards a broad range of pathogens [4]. While the ability of bacteria to develop resistance towards AMPs is debated, MDR bacteria are generally not resistant to AMPs and, in many cases, even show increased sensitivity towards AMPs [5]. Some AMPs, alone or combined with antibiotics, efficiently inhibit biofilm formation and can even disrupt mature biofilms, making them promising antibiofilm agents [6].

TAT-RasGAP $_{317-326}$ is a chimeric peptide consisting of the cellpermeable HIV peptide $\mathrm{TAT}_{48-57}$ linked to a 10-amino acid sequence of the Src Homology 3 Domain (SH3 domain) of p120 RasGAP. TAT-RasGAP ${ }_{317-326}$ was first described for its anticancer properties, being able to sensitise cancer cells to anticancer therapies, directly kill cancer cells and display antimetastatic action [7]. The mechanism of eukaryotic cell killing by this peptide has recently been uncovered. TAT-RasGAP ${ }_{317-326}$ kills cancer cells by first accessing the cytosol through direct translocation across the plasma membrane and then interacting with inner leaflet-enriched phospholipids, such as phosphatidylserine or phosphatidylinositol 4,5-bisphosphate. This interaction eventually lyses the membrane and causes death of the cells [8]. We showed earlier that TATRasGAP $_{317-326}$ can also kill bacteria and that it exerts broad antimicrobial activity both against Gram-positive and Gram-negative human pathogens including $A$. baumannii, $P$. aeruginosa and $S$. aureus [9]. TAT-RasGAP ${ }_{317-326}$ shares properties already described in other AMPs, such as an overall positive charge and several arginine and tryptophan residues. Arginine residues interact with the negatively-charged bacterial membrane, while tryptophan residues appear to insert in the bacterial membrane [10]. These interactions may enable AMPs to translocate into the bacteria without disrupting the membrane and to target intracellular components. While the tryptophan at position 317 of RasGAP domain is essential for the antimicrobial activity of TAT-RasGAP ${ }_{317-326}$ [9], the mode of action of this peptide on prokaryotes remains unknown.

In this report, we questioned the potential effect of TATRasGAP $_{317-326}$ on biofilm formation and mature biofilm expansion. We show that TAT-RasGAP $317-326$ inhibits the formation of $A$. baumannii, $P$. aeruginosa and $S$. aureus biofilms in vitro. Moreover, TATRasGAP $_{317-326}$ reduces the expansion of $A$. baumannii and P. aeruginosa mature biofilms. However, similar to other antibiotics and AMPs, TAT-RasGAP $317-326$ cannot completely eradicate the biofilm scaffold. These results highlight the potential of TAT-RasGAP ${ }_{317-326}$ in the prevention and treatment of biofilms and encourage further development of AMPs as alternative or combinatory antibiofilm treatments.

\section{Materials and methods}

\subsection{Bacterial strains and growth conditions}

Acinetobacter baumannii ATCC 19606 and Staphylococcus aureus ATCC 29213 were obtained from the American Type Culture Collection (ATCC, Manassas, VA, USA). Pseudomonas aeruginosa PA14 was obtained from Prof. Leo Eberl (Department of Plant and Microbial Biology, University of Zürich, Zürich, Switzerland). Bacteria were routinely grown in Mueller-Hinton broth (A. baumannii), tryptic soy broth (S. aureus) or Luria-Bertani broth (P. aeruginosa).

\subsection{Peptides and antibiotics}

TAT-RasGAP ${ }_{317-326}$ is composed of amino acids $48-57$ of the HIV TAT protein (RRRQRRKKRG) and 317-326 of the human RasGAP protein (DTRLNTVWMW) linked with two glycines. TATRasGAP ${ }_{317-326}$ was synthesised as a retro-inverso D-amino acid peptide by SBS Genetech (Beijing, China). Ciprofloxacin, tetracycline and gentamicin were from AppliChem (Darmstadt, Germany), polymyxin B was from Sigma-Aldrich (Saint Louis, MO, USA) and melittin was from Enzo Life Sciences (Farmingdale, NY, USA).

\subsection{Minimum inhibitory concentration (MIC) measurement}

The MICs of antibiotics and AMPs on bacterial strains were determined as described previously [9]. Briefly, overnight cultures were diluted to an optical density at $600 \mathrm{~nm}\left(\mathrm{OD}_{600}\right)$ of 0.1 , were grown for $1 \mathrm{~h}$ at $37^{\circ} \mathrm{C}$ with $200 \mathrm{rpm}$ shaking and were diluted 1:200 in 96-well plates containing increasing amounts of antibiotics or AMPs. Plates were then incubated statically for $18 \mathrm{~h}$ at $37^{\circ} \mathrm{C}$. The lowest concentration at which no turbidity was observed was determined as the MIC.

\subsection{Biofilm formation assay and treatment}

Overnight cultures of bacteria were diluted 1:50 and were grown to exponential phase at $37^{\circ} \mathrm{C}$. Cultures were washed with phosphate-buffered saline (PBS) and adjusted to $\mathrm{OD}_{600}=0.1\left(10^{7}\right.$ $10^{9} \mathrm{CFU} / \mathrm{mL}$ ) in BM2 medium [62 mM potassium phosphate buffer, $7 \mathrm{mM}$ ammonium sulfate, $10 \mu \mathrm{M}$ iron sulfate, $0.4 \%(\mathrm{w} / \mathrm{v})$ glucose, $0.5 \%(\mathrm{w} / \mathrm{v})$ casamino acids, $2 \mathrm{mM}$ magnesium sulfate] with or without antibiotics or AMPs. Then, $100 \mu \mathrm{L}$ of culture was plated in polypropylene plates (Greiner, Kremsmünster, Austria) and biofilms were allowed to form for $24 \mathrm{~h}$ (A. baumannii and $P$. aeruginosa) or $48 \mathrm{~h}$ (S. aureus) at room temperature (RT). For biofilm eradication, mature biofilms were washed twice with PBS and were incubated with antibiotics or AMPs in BM2 medium for 24h at RT.

\subsection{Measurement of biofilm biomass and bacterial viability}

To assess total biofilm biomass, biofilms were washed with water and stained with $0.1 \%$ crystal violet (Sigma-Aldrich) as described previously [11]. Stained biofilms were dried overnight at RT and were dissolved in 30\% acetic acid. Absorbance was measured at $590 \mathrm{~nm}$. To assess bacterial viability, biofilms were washed with PBS and incubated with $4 \mu \mathrm{g} / \mathrm{mL}$ resazurin (Sigma-Aldrich) in BM2. Plates were incubated for $90 \mathrm{~min}$ at $37^{\circ} \mathrm{C}$. Fluorescence was measured with a FLUOstar ${ }^{\circledR}$ Omega Microplate Reader (BMG Labtech, Ortenberg, Germany) with excitation/emission wavelength of $540 / 580 \mathrm{~nm}$.

\subsection{Calculations}

The lowest concentration of antibiotic or AMP that reduced biofilm formation (viability and biomass) by $\geq 90 \%$ was defined as the biofilm prevention concentration $\left(\mathrm{BPC}_{90}\right)$. The minimum biofilm inhibitory concentration (MBIC) was defined as the concentration of antibiotic or AMP that resulted in no expansion of an existing biofilm (viability lower and biomass equal to or lower than the value at treatment). The minimum biofilm eradication concentration $\left(\mathrm{MBEC}_{90}\right)$ was defined as the concentration of antibiotic or AMP that reduced the biofilm initial biomass by $\geq 90 \%$ upon treatment. All calculations were adapted from Macià et al. [12].

\section{Results}

3.1. Classical antibiotics have a moderate effect on Acinetobacter baumannii and Pseudomonas aeruginosa biofilms

Acinetobacter baumannii and P. aeruginosa form biofilms that show increased resistance to antibiotics compared with planktonic bacteria [13]. We first tested whether this observation could be confirmed using our experimental settings by determining the inhibitory potential of antibiotics on planktonic bacteria (MIC) and on bacteria in biofilm formation $\left(\mathrm{BPC}_{90}\right)$. For $A$. baumannii, we 
Table 1

Minimum inhibitory and eradication concentrations of classical antibiotics and antimicrobial peptides (AMPs)

\begin{tabular}{|c|c|c|c|c|}
\hline Antibiotic/AMP & $\operatorname{MIC}(\mu \mathrm{g} / \mathrm{mL})$ & $\mathrm{BPC}_{90}(\mu \mathrm{g} / \mathrm{mL})$ & $\operatorname{MBIC}(\mu \mathrm{g} / \mathrm{mL})$ resazurin/CV a & $\mathrm{MBEC}_{90}(\mu \mathrm{g} / \mathrm{mL})(\% \text { eradication })^{\mathrm{b}}$ \\
\hline \multicolumn{5}{|c|}{ Acinetobacter baumannii ATCC 19606} \\
\hline Ciprofloxacin & 1 & $>256$ & $>256$ & $>256(<10 \%)$ \\
\hline Tetracycline & 1 & $128-256$ & $>256$ & $>256(<10 \%)$ \\
\hline Gentamicin & 32 & $>256$ & $>256$ & $>256(<10 \%)$ \\
\hline TAT-RasGAP $_{317-326}$ & 8 & $16-32$ & $64 / 32$ & $>256(61 \%)$ \\
\hline Polymyxin B & 4 & $8-16$ & $32 / 64$ & $>256(30 \%)$ \\
\hline Melittin & 16 & $16-32$ & $128 / 64$ & $>256(88 \%)$ \\
\hline \multicolumn{5}{|c|}{ Pseudomonas aeruginosa PA14 } \\
\hline Ciprofloxacin & 0.2 & $0.2-0.4$ & $<2 />256$ & $>256(<10 \%)$ \\
\hline Tetracycline & 16 & $4-8$ & $32 />256$ & $>256(<10 \%)$ \\
\hline Gentamicin & 4 & 8 & $32 />256$ & $>256(20 \%)$ \\
\hline TAT-RasGAP $_{317-326}$ & 32 & $16-32$ & 64 & $>256(60 \%)$ \\
\hline Polymyxin B & 2 & 4 & 32 & $>256(65 \%)$ \\
\hline Melittin & 64 & 128 & $>256$ & $>256(<10 \%)$ \\
\hline \multicolumn{5}{|c|}{ Staphylococcus aureus ATCC 29213} \\
\hline Ciprofloxacin & 0.4 & $>256$ & $>256 / 32$ & $>256(57 \%)$ \\
\hline Gentamicin & 8 & $>256$ & $>256 / 64$ & $>256(10 \%)$ \\
\hline TAT-RasGAP $_{317-326}$ & 128 & 128 to $>256$ & $>256$ & $>256(<10 \%)$ \\
\hline Melittin & 32 & 32 & 64 & $>256(<10 \%)$ \\
\hline
\end{tabular}

MIC, minimum inhibitory concentration; BPC, biofilm prevention concentration; MBIC, minimum biofilm inhibitory concentration;

$\mathrm{CV}$, crystal violet; MBEC, minimum biofilm eradication concentration.

a For MBIC, the indication of a single value means that the result was the same with resazurin and CV assays.

b As no $\mathrm{MBEC}_{90}$ could be measured, the percentage of eradication (i.e. decrease in mean $\mathrm{CV} \mathrm{OD}_{590}$ signal) is indicated.

measured a robust increase in bacterial resistance towards the tested antibiotics $\left(\mathrm{BPC}_{90}\right.$ up to $>256$ times the MIC of planktonic bacteria) (Table 1 ). The effect was less striking for $P$. aeruginosa with a $\mathrm{BPC}_{90} / \mathrm{MIC}$ ratio of 2 for ciprofloxacin and gentamicin and 0.25 for tetracycline (Table 1 ).

In a further step, we tested whether these antibiotics could limit biofilm expansion (measuring the MBIC) and eradicate established biofilms (measuring the $\mathrm{MBEC}_{90}$ ). We used the reduction of resazurin to resorufin as a surrogate of bacterial viability and measured total biomass with crystal violet staining. All of the tested antibiotics were ineffective both at inhibiting expansion and eradicating $A$. baumannii biofilms (MBIC and $\mathrm{MBEC}_{90},>256 \mu \mathrm{g} / \mathrm{mL}$ ) (Table 1). While ciprofloxacin, tetracycline and gentamicin limited the proliferation of bacteria in $P$. aeruginosa biofilms at concentrations corresponding to 2-4 times the $\mathrm{BPC}_{90}$, we observed no effect on biomass (Table 1). These results confirm the low efficiency of classical antibiotics on biofilms.

3.2. TAT-RasGAP $317-326$ potently inhibits the formation and expansion of Acinetobacter baumannii and Pseudomonas aeruginosa biofilms

A number of AMPs and their derivatives were reported to reduce biofilm formation and to degrade existing bacterial biofilms, highlighting an antibiofilm effect for AMPs [14]. Using the model AMPs melittin and polymyxin B, we observed a consistent inhibitory effect on $A$. baumannii and $P$. aeruginosa biofilm formation. The $\mathrm{BPC}_{90}$ of melittin was equal to twice the MIC for A. baumannii biofilms and 2-4 times higher than the MIC for $P$. aeruginosa biofilms (Table 1 ). For polymyxin $\mathrm{B}$, the $\mathrm{BPC}_{90} / \mathrm{MIC}$ ratios were $2-4$ and 2 for $A$. baumannii and $P$. aeruginosa, respectively (Table 1 ). As TAT-RasGAP $317-326$ is efficient against planktonic $A$. baumannii and $P$. aeruginosa [9], we hypothesised that it would also potently inhibit the formation of biofilms. TAT-RasGAP ${ }_{317-326}$ inhibited A. baumannii biofilm formation at $16-32 \mu \mathrm{g} / \mathrm{mL}$, corresponding to $1-2$ times the MIC (Fig. 1; Table 1). The BPC $_{90}$ of TAT-RasGAP ${ }_{317-326}$ on $P$. aeruginosa biofilms was equal to its MIC (32 $\mu \mathrm{g} / \mathrm{mL}$ ) (Fig. 1 ; Table 1).

Since classical antibiotics had little to no effect on established biofilms (Table 1), we tested the ability of AMPs to inhibit the expansion and to eradicate established biofilms. TAT-RasGAP ${ }_{317-326}$ limited the expansion of $A$. baumannii biofilms with a MBIC/MIC ratio of 4-8 (Fig. 2; Table 1). Melittin was effective at 4-8 times the MIC, while polymyxin $\mathrm{B}$ had an MBIC/MIC ratio of 8-16 (Table 1). TAT-RasGAP ${ }_{317-326}$ efficiently inhibited $P$. aeruginosa biofilm expansion with an MBIC/MIC ratio of 2 (Fig. 2; Table 1). In contrast, polymyxin $\mathrm{B}$ had a higher MBIC/MIC ratio of 16 , while the MBIC of melittin could not be determined, being $>256 \mu \mathrm{g} / \mathrm{mL}$ (Table 1). None of the tested AMPs eradicated $>90 \%$ of the biomass of $A$. baumannii and $P$. aeruginosa biofilms. However, they still induced a stronger biomass reduction at the maximum tested concentration of $256 \mu \mathrm{g} / \mathrm{mL}$ compared with antibiotics (Table 1 ).

\subsection{TAT-RasGAP $317-326$ inhibits the formation of Staphylococcus aureus biofilms}

TAT-RasGAP $317-326$ is effective both on Gram-negative and Gram-positive bacteria in planktonic cultures [9]. We thus tested its impact on $S$. aureus biofilms. Despite the high MIC of TATRasGAP $_{317-326}$ on planktonic S. aureus $(128 \mu \mathrm{g} / \mathrm{mL})$, we could measure a $\mathrm{BPC}_{90} / \mathrm{MIC}$ ratio of $1-2$ by resazurin reduction (Table 1 ; Fig. 1). In comparison, the $\mathrm{BPC}_{90} / \mathrm{MIC}$ ratio of melittin was 0.51 , while none of the tested antibiotic prevented biofilm formation $\left(\right.$ BPC $_{90}>256 \mu \mathrm{g} / \mathrm{mL}$ ) (Table 1 ). However, the MBIC and $\mathrm{MBEC}_{90}$ of TAT-RasGAP $317-326$ on $S$. aureus biofilm could not be estimated, being $>256 \mu \mathrm{g} / \mathrm{mL}$. It has to be noted that $S$. aureus formed apparently weaker biofilms than $P$. aeruginosa and A. baumannii (staining with crystal violet being strongly reduced) (Fig. 1B; Fig. 2B) leading to high variability in the results.

\section{Discussion}

Bacterial biofilms cause nosocomial infections and underlie several chronic infections. The complex structure of biofilms renders them refractory to treatments with classical antibiotics. Here we show that the AMP TAT-RasGAP ${ }_{317-326}$ has a potent inhibitory effect on $A$. baumannii, $P$. aeruginosa and, to a lesser extent, $S$. aureus biofilm formation and expansion. These observations support the potential use of AMPs as alternatives to classical antibiotics in antibiofilm treatment.

To test the quality of the biofilm produced in vitro, we measured its resistance to classical antibiotics. As reported in the literature, we observed increased resistance of $A$. baumannii and $P$. 

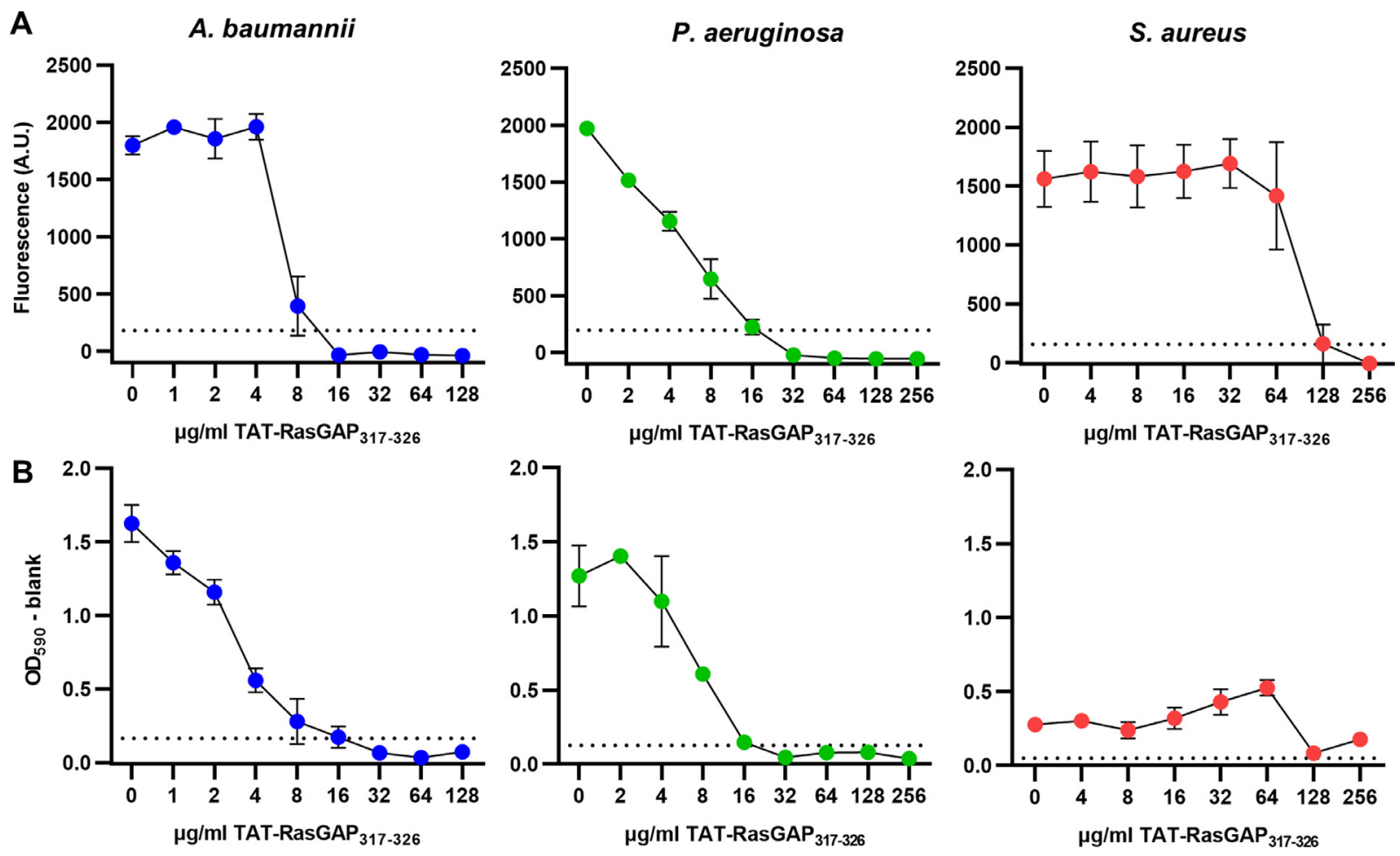

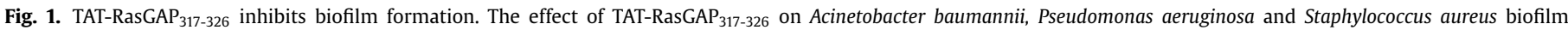

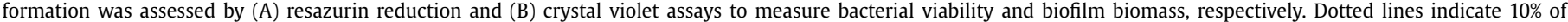
initial signal. Data are the mean \pm SEM of three experiments performed in quadruplicate.
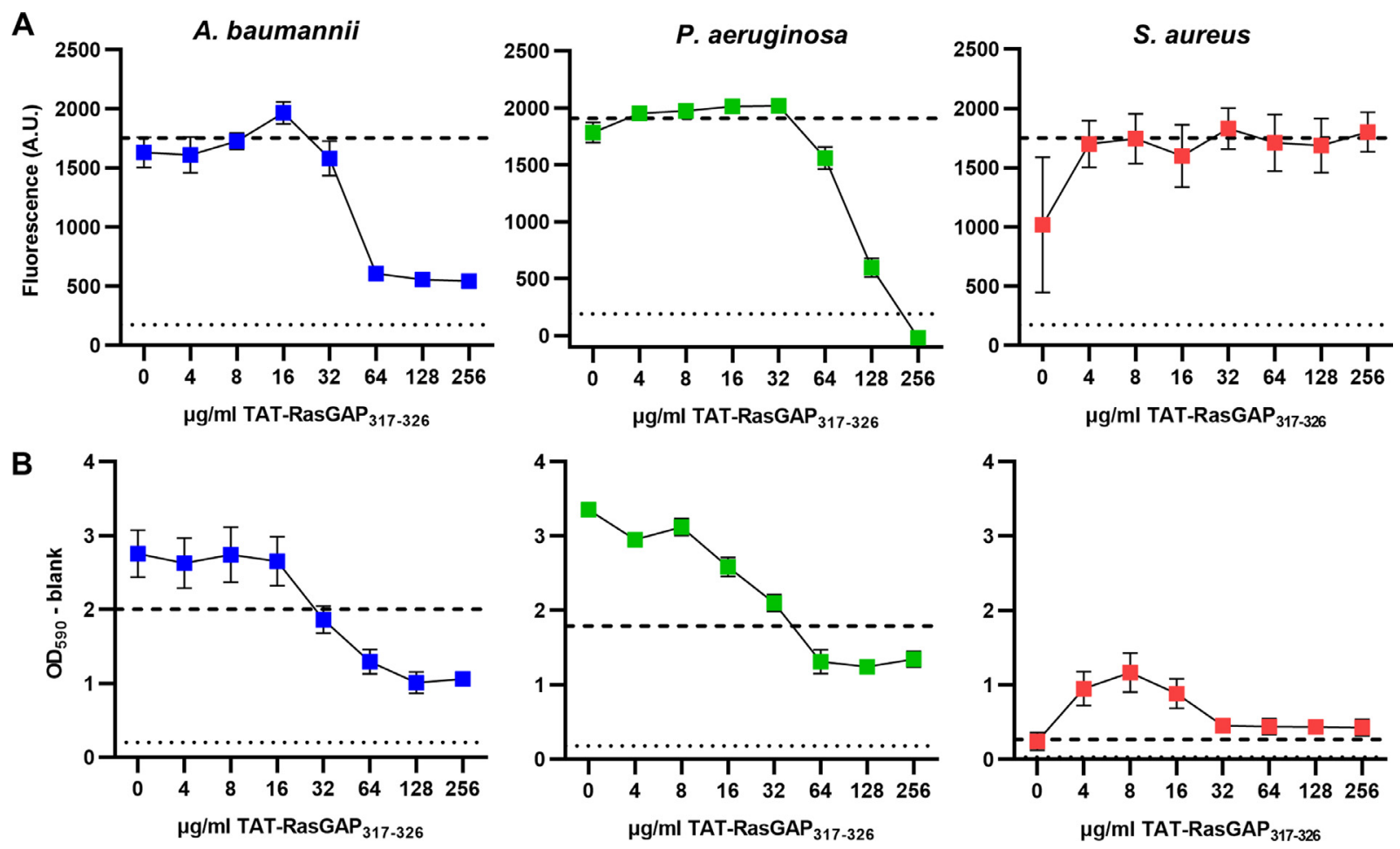

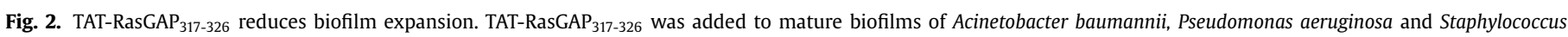

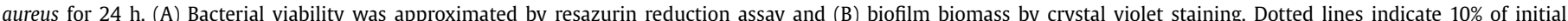
signal. Dashed lines indicate values for established biofilms before treatment. Data are the mean \pm SEM of three experiments performed in quadruplicate. 
aeruginosa biofilms to classical antibiotics compared with planktonic cultures. However, resistance was higher for $A$. baumannii biofilms (up to $>512$ times the MIC) than for $P$. aeruginosa biofilms (2-4 times the MIC). This difference in biofilm resistance to antibiotics is possibly caused by a faster resistance development during $A$. baumannii biofilm formation, partly triggered by the antibiotics themselves [15]. While antibiotics showed some effect on biofilm formation at moderate to high concentrations, they were inefficient in inhibiting proliferation or disrupting mature biofilms (Table 1), as reported previously [16]. This low efficiency of antibiotics on biofilms highlights the need for alternative strategies to target these biofilms.

In contrast to antibiotics, the TAT-RasGAP $317-326$, polymyxin $\mathrm{B}$ and melittin AMPs limited both bacterial biofilm formation and expansion. Moreover, they showed a stronger biofilm eradication potential compared with antibiotics. Polymyxin B and melittin were already reported to have potent antibiofilm activity against A. baumannii and $P$. aeruginosa [17,18]. Our results further support the use of AMPs alone or in combination with other drugs as a possible alternative for the treatment of biofilm-associated infections. The combination of membrane-targeting AMPs with classical antibiotics may be a valuable way to eradicate biofilmembedded bacteria. In addition to directly killing bacterial cells, AMPs may also interact with biofilm matrix component or signalling molecules, thus weakening the biofilm scaffold and promoting antibiotic entry. In line, the combination of melittin and LL-37 with classical antibiotics reduced the corresponding MBEC by several fold [19]. In clinical settings, several studies reported the combination of polymyxin $B$ with classical antibiotics for the treatment of MDR bacterial infections, including chronic possibly biofilm-mediated infections, with promising results [20]. In future studies, we would like to test the antibiofilm effect of combinations of TAT-RasGAP ${ }_{317-326}$ with classical antibiotics. Indeed, TATRasGAP $_{317-326}$ might have advantages compared with other AMPs: its low toxicity to mammalian cells [9], and its chimeric and synthetic nature that should avoid selection of pre-existing resistance and lower the risk of immune response alteration. Moreover, since biofilms are often composed of multiple bacterial species, the broad-spectrum antimicrobial activity of TAT-RasGAP ${ }_{317-326}$ is also an attractive feature. While the current version of TATRasGAP $_{317-326}$ showed low bioavailability [9], it could have a potent effect on the treatment of biofilm-associated infections when administrated locally or as implant-covering agent. Furthermore, potential modifications such as addition of positive charges, addition of lipid moieties, or amino acid modifications might improve the bioavailability of this peptide and thus increase its efficiency. Nevertheless, combinations of TAT-RasGAP ${ }_{317-326}$ with classical antibiotics or other AMPs might cause synergism against biofilms and could lead to development of combinatory treatments.

In summary, we show that the AMP TAT-RasGAP ${ }_{317-326}$ has potent antibiofilm activity in vitro against $A$. baumannii and $P$. aeruginosa and, to a lesser extent, $S$. aureus biofilms. This makes TAT-RasGAP ${ }_{317-326}$ a promising tool in the treatment of biofilmassociated infections alone or in combination with other antimicrobial agents.

\section{Competing interests}

None declared.

\section{Acknowledgments}

The authors thank Prof. Gilbert Greub for providing laboratory equipment, Prof. Nibbering's group (Leiden, Netherlands) for their assistance in implementation of the protocol of in vitro forma- tion of biofilms, and Prof. Eberl (Zurich, Switzerland) for providing strains.

\section{Funding}

$\mathrm{NJ}$ and CW received an interdisciplinary grant from the University of Lausanne (Lausanne, Switzerland).

\section{Ethical approval}

Not required.

\section{References}

[1] Cassini A, Diaz Högberg L, Plachouras D, Quattrocchi A, Hoxha A, Skov Simonsen $G$, et al. Attributable deaths and disability-adjusted life-years caused by infections with antibiotic-resistant bacteria in the EU and the European Economic Area in 2015: a population-level modelling analysis. Lancet Infect Dis 2019;19:56-66.

[2] Flemming HC, Wingender J, Szewzyk U, Steinberg P, Rice SA, Kjelleberg S. Biofilms: an emergent form of bacterial life. Nat Rev Microbiol 2016; 14:563-75.

[3] Lebeaux D, Ghigo JM, Beloin C. Biofilm-related infections: bridging the gap between clinical management and fundamental aspects of recalcitrance toward antibiotics. Microbiol Mol Biol Rev 2014;78:510-43.

[4] Bahar AA, Ren D. Antimicrobial peptides. Pharmaceuticals (Basel) 2013;6:1543-75.

[5] Lázár V, Martins A, Spohn R, Daruka L, Grézal G, Fekete G, et al. Antibiotic-resistant bacteria show widespread collateral sensitivity to antimicrobial peptides. Nat Microbiol 2018;3:718-31.

[6] Mulani MS, Kamble EE, Kumkar SN, Tawre MS, Pardesi KR. Emerging strategies to combat ESKAPE pathogens in the era of antimicrobial resistance: a review. Front Microbiol 2019;10:539.

[7] Heulot M, Chevalier N, Puyal J, Margue C, Michel S, Kreis S, et al. The TAT-RasGAP ${ }_{317-326}$ anti-cancer peptide can kill in a caspase-, apoptosis-, and necroptosis-independent manner. Oncotarget 2016;7:64342-59.

[8] Serulla M, Ichim GF, Stojceski F, Grasso G, Afonin S, Heulot M, et al. TAT-RasGAP $317-326$ kills cells by targeting inner-leaflet-enriched phospholipids. Proc Natl Acad Sci U S A 2020;117:31871-81.

[9] Heulot M, Jacquier N, Aeby S, Le Roy D, Roger T, Trofimenko E, et al. The anticancer peptide TAT-RasGAP $317-326$ exerts broad antimicrobial activity. Front Microbiol 2017:8:994.

[10] Mishra AK, Jaehyuk Choi, Moon E, Baek K-H. Tryptophan-rich and proline-rich antimicrobial peptides. Molecules 2018;23:815.

[11] de Breij A, Riool M, Cordfunke RA, Malanovic N, de Boer L, Koning RI, et al. The antimicrobial peptide SAAP-148 combats drug-resistant bacteria and biofilms. Sci Transl Med 2018;10:eaan4044 Erratum in: Sci Transl Med 2018; 10:eaat5731.

[12] Macià MD, Rojo-Molinero E, Oliver A. Antimicrobial susceptibility testing in biofilm-growing bacteria. Clin Microbiol Infect 2014;20:981-90.

[13] Sanchez CJ Jr, Mende K, Beckius ML, Akers KS, Romano DR, Wenke JC, et al. Biofilm formation by clinical isolates and the implications in chronic infections. BMC Infect Dis 2013:13:47.

[14] Fleming D, Rumbaugh KP. Approaches to dispersing medical biofilms. Microorganisms 2017;5:15

[15] Penesyan A, Nagy SS, Kjelleberg S, Gillings MR, Paulsen IT. Rapid microevolution of biofilm cells in response to antibiotics. NPJ Biofilms Microbiomes 2019;5:34.

[16] Olson ME, Ceri H, Morck DW, Buret AG, Read RR. Biofilm bacteria: formation and comparative susceptibility to antibiotics. Can J Vet Res 2002;66:86-92.

[17] Beganovic M, Luther MK, Daffinee KE, LaPlante KL. Biofilm prevention concentrations (BPC) of minocycline compared to polymyxin B, meropenem, and amikacin against Acinetobacter baumannii. Diagn Microbiol Infect Dis 2019;94:223-6.

[18] Picoli T, Mendes Peter C, Zani Luíz J, Bressan Waller S, Gomes Lopes M, Neutzling Boesche $\mathrm{K}$, et al. Melittin and its potential in the destruction and inhibition of the biofilm formation by Staphylococcus aureus, Escherichia coli and Pseudomonas aeruginosa isolated from bovine milk. Microb Pathog 2017; 112:57-62.

[19] Dosler S, Karaaslan E. Inhibition and destruction of Pseudomonas aeruginosa biofilms by antibiotics and antimicrobial peptides. Peptides 2014;62:32-7.

[20] Mattos KPH, Gouvêa IR, Quintanilha JCF, Cursino MA, Vasconcelos PENS, Moriel P. Polymyxin B clinical outcomes: a prospective study of patients undergoing intravenous treatment. J Clin Pharm Ther 2019;44:415-19. 